\title{
Composition of Myrtus communis L. Essential Oils as Affected by Climatic Conditions
}

\author{
Composição dos Óleos Essenciais de Myrtus communis L. Influenciada por Condições \\ Climáticas
}

\author{
Paula Pereiraa $^{1,2}$, Maria João Cebola ${ }^{1,2,3}$, M. Gabriela Bernardo-Gil ${ }^{2}$ \\ ${ }^{1}$ CBIOS - Universidade Lusófona's Research Centre for Biosciences and Health Technologies, ULHT, Av. Campo Grande, \\ 376, 1749-024 Lisboa, Portugal \\ ${ }^{2}$ CERENA - Centre for Natural Resources and the Environment, Instituto Superior Técnico, Universidade de Lisboa, Av. \\ Rovisco Pais, 1049-001 Lisboa, Portugal \\ ${ }^{3}$ ENIDH - Escola Superior Náutica Infante D. Henrique, Av. Eng. Boneville Franco, 2770-058 Paço de Arcos, Portugal \\ Email: mjoaocebola@enautica.pt
}

\begin{abstract}
Myrtus Communis L. (Myrtaceae family), commonly known as myrtle, possesses a set of qualities that make it very interesting for the pharmaceutical, nutraceutical and cosmetic industries.

In this work, myrtle was studied over a period of three years (2006-2008), encompassing the main stages of the development cycle of the plant. The influence of climatic conditions, such as temperature and rainfall, on the chemical composition and yield of the essential oils obtained from leaves and berries was studied. The leaves and berries essential oils were obtained by Clevenger distillation and analysed by GC and GC-MS. The results show that the major components were limonene+1,8-cineole, myrtenyl acetate, $\alpha$-pinene and linalool. Over the three year period of study, a decrease in the composition of the most volatile compounds ( $\alpha$-pinene and limonene $+1,8$ cineole) was observed, the reverse being found for linalool and myrtenyl acetate. The highest value for the yield of the leaves' essential oils was observed in the third year $(0.64 \%, \mathrm{w} / \mathrm{w})$, whereas that same year the lowest yield $(0.07 \%, \mathrm{w} / \mathrm{w})$ of fruit was observed. These results were likely due to an atypically high record for rainfall in April and May of that year. The highest value of the berry essential oils $(0.14 \%, \mathrm{w} / \mathrm{w})$ was obtained in the second year.
\end{abstract}

Keywords: Myrtle, Myrtus communis L., Essential oils, composition, climate.

\section{Resumo}

Myrtus communis L. (família das Mirtáceas), vulgarmente conhecida como murta, possui um conjunto de propriedades que a tornam muito interessante para as indústrias farmacêutica, nutracêutica e de cosméticos.

Neste trabalho, estudou-se a murta ao longo de um período de três anos (2006-2008), de modo a abranger as principais fases do ciclo vegetativo da planta. Estudou-se a influência das condições climáticas, tais como a temperatura e a precipitação, na composição química dos óleos essenciais e no rendimento das folhas e dos frutos. Os óleos essenciais das folhas e dos frutos foram obtidos por destilação do tipo Clevenger e analisados por GC e GC-MS. Os resultados mostram que os principais componentes foram o limoneno $+1,8$-cineol, o acetato de mirtenilo, o $\alpha$-pineno e o linalol. Ao longo do período de três anos de estudo, observou-se um decréscimo na composição dos compostos mais voláteis ( $\alpha$-pineno e limoneno+1,8-cineol), sendo o inverso encontrado para o linalol e o acetato de mirtenilo. $\mathrm{O}$ valor mais elevado do rendimento dos óleos essenciais foi obtido para as folhas, no terceiro ano $(0,64 \%, \mathrm{~m} / \mathrm{m})$, enquanto que nesse ano se observou o menor rendimento $(0,07 \%, \mathrm{~m} / \mathrm{m})$ para os frutos. Estes resultados foram, provavelmente, devido ao fato de ter sido registado um nível de precipitação atipicamente elevado no período entre abril e maio do mesmo ano. O valor mais elevado dos óleos essenciais dos frutos $(0,14 \%, \mathrm{~m} / \mathrm{m})$ foi obtido no segundo ano.

Palavras-chave: Murta, Myrtus Communis L., óleos essenciais, composição, clima 


\section{Introduction}

Myrtle (Myrtus communis L.), is a common sight in the woods of most Mediterranean countries. Portugal is no exception, and this shrub can be found growing wild in most of its continental area with the exception of the far northern interior part of the country. Myrtle is an evergreen shrub with very aromatic dark green leaves, white, delicate flowers and small, round, dark blue/purple fruit, having high essential oils content in its leaf, flower and fruit glands [1]. Its pleasant scent makes it a valued ingredient for the perfume and cosmetic industries. Its flavour makes it an interesting spice for culinary purposes, although this particular aspect has been neglected in most countries with only a few exceptions. In Portugal myrtle is mainly used to make regional liqueurs. As a member of the aromatic plants and being typical of the Mediterranean flora, myrtle has been the object of a number of studies which have shown that it also possesses very interesting medicinal properties, being referred as an antiseptic, antimicrobial, disinfectant and hypoglycemic agent [2-3].

Previous studies have shown that some differences arise in the composition of the essential oils due to the different stages of the vegetative cycle of the plant and also due to growth in different geographical locations [4-5]. In an earlier study, we had ascertained that the essential oils from Portuguese myrtle are characterized by the presence of myrtenyl acetate as its major component [4].

The present work looks at the variation of the yield and composition of essential oils over a three year period, exploring the possible influence of the climatic conditions on these variables. The climatic factors investigated were precipitation and temperature, as shrubs found in the wild such as myrtle are often exposed to extreme weather conditions including drought and excess UV-B radiation in the summer months. Production of essential oils is known to be triggered as part of a response mechanism to stress. It has been reported that environmental factors play a key role in the chemical composition of myrtle oils because of their influence on the plant biosynthetic pathways [6-7]. For example, the low temperatures (cold and frost) occurring in winter, during the plant's dormant stage, can have profound negative impacts on plants [8-10].

\section{Introdução}

Murta (Myrtus communis L.), é parte integrante da maioria das matas dos países mediterrânicos. Portugal não é excepção e este arbusto pode ser encontrado nas matas de praticamente todo o território nacional, com exceção da parte interior norte do país.

A murta é um arbusto perene com folhas verdes escuras, muito aromáticas, delicadas flores brancas e pequenos frutos, em forma de baga, de cor azul arroxeada. Exibe um alto teor dos óleos essenciais nas glândulas das suas folhas, flores e frutos [1].

O seu agradável aroma torna-a num valioso ingrediente para as indústrias de perfumes e cosméticos. $\mathrm{O}$ seu sabor torna-a um tempero interessante para fins culinários, embora este aspecto em particular, tenha sido negligenciado na maioria dos países, com apenas algumas exceções. Em Portugal a murta é utilizada principalmente para licores regionais. A Murta é uma planta aromática e dado ser típica da flora do Mediterrâneo, tem sido objeto de um número de estudos que demonstraram que este arbusto possui propriedades medicinais muito interessantes e tem sido referida como antisséptica, antimicrobiana, desinfetante e agente hipoglicémico [2-3]. Estudos anteriores mostraram que algumas diferenças surgem na composição dos óleos essenciais devido às diferentes fases do ciclo vegetativo da planta e também devido a diferentes localizações geográficas [4-5]. Já tínhamos concluído, num estudo anterior [4],que os óleos essenciais da murta Portuguesa são caracterizados por terem o acetato de mirtenilo como o seu principal componente

O presente trabalho estuda a variação do rendimento dos óleos essenciais e composição ao longo de um período de três anos, explorando a possível influência das condições climáticas sobre estas variáveis. Os fatores climáticos investigados foram a precipitação e a temperatura, dado que os arbustos silvestres como a murta se encontram, muitas vezes, expostos a condições climáticas extremas, como a seca e a radiação excessiva de UV (ultra violeta) nos meses de verão. Sabe-se que a produção dos óleos essenciais é desencadeada como parte de um mecanismo de resposta ao stress. Scora (1973) e Akin et al. (2010), verificaram que os fatores ambientais desempenham um papel fundamental na composição química dos óleos essenciais de murta devido à sua influência sobre as vias de biossíntese das plantas. Por exemplo, as baixas temperaturas (frio e geada) que ocorrem no inverno, durante a fase de dormência da planta, têm profundos impactos negativos sobre as plantas [8-10]. 


\section{Material and methods}

\section{Plant Material}

Samples of myrtle leaves and berries were collected over a period of three years (2006-2008) from the Sintra area of Portugal. For each year, the samples studied were, for the leaves, collected in July (flowering stage) and, for the fruit, in October (ripening stage) [4]. No soil tillage, fertilization or pest treatments were provided in this area. Collection was consistently made in the same geographic area to avoid variability. After collection, the plant material was identified and deposited in the Herbarium of the Superior Agronomy Institute of the Technical University of Lisbon (LISI 457/2006; LISI 873/2006). The plant material retained for analysis was dried for two months out of the sunlight, and then sealed in black plastic bags and kept at $-20^{\circ} \mathrm{C}$.

\section{Standards and reagents}

GC-MS: $\alpha$-pinene, $\beta$-pinene, myrcene, $\alpha$-terpinene, p-cymene, limonene, 1,8-Cineole, $\gamma$-terpinene, linalool oxide, linalool, fenchol, borneol, terpinen4-ol, $\alpha$-terpineol , myrtenol, geraniol, borneol acetate, myrtenyl acetate, neryl acetate, geranyl acetate, $\beta$-caryophyllene, $\alpha$-humulene, caryophyllene oxide, alkane standard solutions $\left(\mathrm{C}_{8}-\mathrm{C}_{20}\right)$ and $\left(\mathrm{C}_{21}-\mathrm{C}_{40}\right)$ were acquired from Aldrich (Germany, UK), Fluka (Switerland) and Extrasynthese (France).

\section{Extraction Procedure}

The essential oils were obtained by hydrodistillation using a Clevenger-type apparatus, for two hours, using $100 \mathrm{~g}$ of ground dried plant material. The oils were recovered, weighed, frozen, and stored in a sealed flask at $-20^{\circ} \mathrm{C}$ until GC-MS analysis was performed.

\section{GC-FID Analysis}

All the samples were analysed with at least three replicates. The analyses were carried out on a Hewlett-Packard 5890 Series II gas chromatograph equipped with a FID detector (with a supply of high purity air, $\mathrm{P}=6$ bar, and hydrogen, $\mathrm{P}=1$ bar. The capillary column used was an HP-5 cross-linked 5\% (composition 5\% diphenyl, $95 \%$ dimethylpolysiloxane with $50 \mathrm{~m}$ length, $0.32 \mathrm{~mm}$ i.d. and $0.17 \mu \mathrm{m}$ film thickness). The injector and detector were operated at $200{ }^{\circ} \mathrm{C}$ and $250{ }^{\circ} \mathrm{C}$, respectively. Split mode was used at a ratio of 1:20. The oven temperature was programmed as follows: $60^{\circ} \mathrm{C}$ for 10 min, increased up to $180{ }^{\circ} \mathrm{C}$ at $2^{\circ} \mathrm{C} / \mathrm{min}$ and held isothermally for $10 \mathrm{~min}$. Nitrogen was used as the carrier gas $(\mathrm{P}=3$ bar). Samples of $0.1 \mu 1$ were injected with a

\section{Materiais e Métodos}

\section{Material Vegetal}

As amostras de folhas e frutos de murta foram recolhidas no concelho de Sintra (Portugal) durante um período de três anos (2006-2008). Para cada ano, as amostras estudadas foram recolhidas, as folhas em julho, (fase de floração) e os frutos em outubro (fase de amadurecimento) [4]. O solo não foi submetido a nenhum tipo de preparação quer em termos de fertilização, quer em termos de tratamento de pragas. A recolha teve sempre lugar na mesma área geográfica para evitar variabilidade. Após a recolha, a planta foi identificada e depositada no Herbário do Instituto Superior de Agronomia da Universidade Técnica de Lisboa (LISI 457/2006; LISI $873 / 2006$ ). A planta foi seca durante dois meses, protegida da luz, e em seguida foi selada e armazenada a $-20{ }^{\circ} \mathrm{C}$.

\section{Padrões e reagentes}

GC-MS: $\alpha$-pineno, $\beta$-pineno, mirceno, $\alpha$-terpineno, p-cimeno, limoneno, 1,8-Cineol, $\gamma$-terpineno, óxido de linalol, linalol, fenchol, borneol, terpineno-4-ol, $\alpha$-terpineol , mirtenol, geraniol, acetato de borneol, acetato de mirtenilo, acetato de nerilo, acetato de geranilo, $\beta$-cariofileno, $\alpha$-humuleno, oxido de cariofileno, soluções padrão de alcanos $\left(\mathrm{C}_{8}-\mathrm{C}_{20}\right)$ e $\left(\mathrm{C}_{21}-\mathrm{C}_{40}\right)$ foram adquiridas na Aldrich (Alemanha, Reino Unido), Fluka (Suiça) e Extrasynthese (França).

\section{Método de Extração}

Os óleos essenciais foram obtidos por hidrodestilação de $100 \mathrm{~g}$ de planta, utilizando o destilador de Clevenger, durante duas horas. Os óleos essenciais obtidos foram pesados, congelados e armazenados em frascos selados a $-20^{\circ} \mathrm{C}$ até à realização da análise por GC-MS.

\section{Análise por GC-FID}

As amostras foram analisadas em três réplicas. As análises foram realizadas num cromatógrafo gasoso 5890 Series II da Hewlett-Packard equipado com um detetor por ionização em chama (FID) (abastecido com ar, $\mathrm{P}=6$ bar, e hidrogénio, $\mathrm{P}=1$ bar, de elevado grau de pureza). A coluna capilar utilizada foi uma HP-5 reticulada 5\% (composição 5\% difenil, 95\% de dimetilpolissiloxano com $50 \mathrm{~m}$ de comprimento, $0,32 \mathrm{~mm} \mathrm{~d}_{\mathrm{i}}$ e $0,17 \mu \mathrm{m}$ de espessura do filme). $\mathrm{O}$ injetor e detetor funcionaram a $200{ }^{\circ} \mathrm{C}$ e $250{ }^{\circ} \mathrm{C}$, respetivamente. Utilizou-se um modo Split com uma proporção de 1:20. A temperatura do forno foi programada do seguinte modo: $60^{\circ} \mathrm{C}$ durante 10 minutos, aumentou-se para $180{ }^{\circ} \mathrm{C}$ a $2{ }^{\circ} \mathrm{C} \mathrm{min}{ }^{-1}$ e foi 
$1 \mu 1$ micro-syringe (Hamilton). The essential oils samples were injected without dilution. The linear retention indices were determined using a homologous series of n-alkanes $\left(\mathrm{C}_{9}\right.$ to $\left.\mathrm{C}_{25}\right)$. The components were identified by comparison with the linear retention index (LRI) of standard components and by comparison with the LRI published in the literature [11-15].

\section{GC-MS Analysis}

The results of GC were confirmed by GC-MS. The analyses were carried out on a trace GC ultra and Trace DSQ (Thermo). The capillary column was a TR-5MS (30 m length, $0.5 \mathrm{~mm}$ i.d. and $0.5 \mu \mathrm{m}$ film thickness). The injector and the interface were operated at $200^{\circ} \mathrm{C}$ and $250^{\circ} \mathrm{C}$ respectively. Helium was the carrier gas with a flow rate of $1 \mathrm{ml} / \mathrm{min}$. Split mode was used at a ratio of 1:30. MS conditions were as follows: ionization voltage, $70 \mathrm{eV}$; full rate; mass range 50-420 amu; ion source temperature $260^{\circ} \mathrm{C}$. The oven temperature program was as follows: $60^{\circ} \mathrm{C}$ for $10 \mathrm{~min}$, increased at $2^{\circ} \mathrm{C} / \mathrm{min}$ to $180^{\circ} \mathrm{C}$, increased at $10^{\circ} \mathrm{C} / \mathrm{min}$ to $200^{\circ} \mathrm{C}$, held isothermally for $30 \mathrm{~min}$ and increased at $10^{\circ} \mathrm{C} /$ min to $240{ }^{\circ} \mathrm{C}$. The compounds were identified through library search using Wiley GC/MS Library, Mistdemo and MainLib.

\section{Statistical analysis}

All data were expressed as the mean \pm SD (standard deviation) of triplicate experiments, and both one-way, and two-way analyses of variance were applied.

\section{Results and Discussion}

\section{Extraction yield}

The essential oils obtained, both from the leaves and from the fruits, had a fresh and pleasant odour and a light yellow colour. The average yield obtained on the basis of three successive extractions is presented in Table 1 . This table shows that the highest essential oils yield was obtained from the leaves in the year 2008. In contrast, this was the year in which the lowest yield was obtained for the fruit essential oils. Climatic conditions are known to exert a strong influence on fruit growth. Figure 1 shows the climatic data for the three years under study. Some differences can be observed within mantida isotermicamente durante $10 \mathrm{~min}$. Utilizou-se azoto como gás de arraste $\left(\mathrm{P}\left(\mathrm{N}_{2}\right)=3\right.$ bar).

As amostras dos óleos essenciais, $0,1 \mu \mathrm{L}$, foram injetadas sem diluição com uma micro-seringa Hamilton de $1 \mu \mathrm{L}$. Os índices de retenção linear foram determinados utilizando uma série homóloga de $n$-alcanos $\left(\mathrm{C}_{9}\right.$ to $\left.\mathrm{C}_{25}\right)$. Os compostos foram identificados por comparação com os índices de retenção linear (LRI) dos padrões e por comparação com os LRI publicados na literatura [11$15]$.

\section{Análise por GC-MS}

Os resultados de GC foram confirmados por GC-MS. As análises foram realizadas num cromatógrafo gasoso Trace GC Ultra eTrace DSQ - Thermo. A coluna capilar foi uma TR-5MS (30m de comprimento, $0,25 \mathrm{~mm} \mathrm{~d}_{i}$ e espessura de filme de $0,25 \mu \mathrm{m})$. $\mathrm{O}$ injetor e a interface funcionaram a $200^{\circ} \mathrm{C}$ e $250^{\circ} \mathrm{C}$, respetivamente. $\mathrm{O}$ hélio foi o gás de arraste com um caudal de $1 \mathrm{~mL} \mathrm{min-}$ ${ }^{1}$. Utilizou-se um modo ,split“ com uma proporção de 1:30. As condições de MS foram as seguintes: tensão de ionização, $70 \mathrm{eV}$; débito máximo; alcance mássico 50 a 420 amu; temperatura da fonte iónica $260^{\circ} \mathrm{C}$. O programa de temperatura do forno foi o seguinte: $60^{\circ} \mathrm{C}$ durante $10 \mathrm{~min}$, subida de temperatura para $180^{\circ} \mathrm{C}$ a $2^{\circ} \mathrm{C}$ $\mathrm{min}^{-1}$, subida de temperatura para $200^{\circ} \mathrm{C}$ a $10^{\circ} \mathrm{C} \min ^{-1}$; a temperatura manteve-se constante a $200^{\circ} \mathrm{C}$ durante 30 min após o que foi aumentada para $240^{\circ} \mathrm{C} \mathrm{a} 10^{\circ} \mathrm{C} \mathrm{min}^{-1}$. Os compostos foram identificados por pesquisa bibliográfica utilizando a Biblioteca Wiley GC MS, Mistdemo e MainLib.

\section{Análise Estatística}

Todos os ensaios foram realizados em triplicado e foram expressos como média \pm desvio padrão. Realizou-se a análise estatística, ANOVA, dois fatores e um fator

\section{Resultados e Discussão}

\section{Rendimento de Extração}

Os óleos essenciais obtidos, tanto para as folhas como para o fruto, apresentavam um cheiro fresco e agradável e uma coloração amarelo pálido. Os rendimentos médios obtidos com base em três extrações sucessivas são apresentados na Tabela 1. Esta mostra que o valor mais elevado de rendimento dos óleos essenciais foi obtido para as folhas, no ano de 2008, obervando-se, nesse mesmo ano, o menor rendimento dos óleos essenciais para os frutos. Sabe-se que as condições climáticas exercem uma forte influência no crescimento dos frutos. 
Table 1/ Tabela 1 - Essential oils yield for the three years studied.

Rendimento dos óleos essenciais para os três anos de colheita.

\begin{tabular}{|c|c|c|c|}
\hline \multirow[t]{2}{*}{ Plant Part / Parte da Planta } & \multicolumn{3}{|c|}{$\begin{array}{l}\text { Yield / Rendimento } \\
\text { (g/100g dried plant / planta seca) }\end{array}$} \\
\hline & 2006 & 2007 & 2008 \\
\hline Leaves/Folhas (July/julho) & $0,48 \pm 0,01$ & $0,48 \pm 0,01$ & $0,64 \pm 0,01$ \\
\hline Berries/Frutos(October/outubro) & $0,11 \pm 0,06$ & $0,14 \pm 0,02$ & $0,07 \pm 0,01$ \\
\hline
\end{tabular}

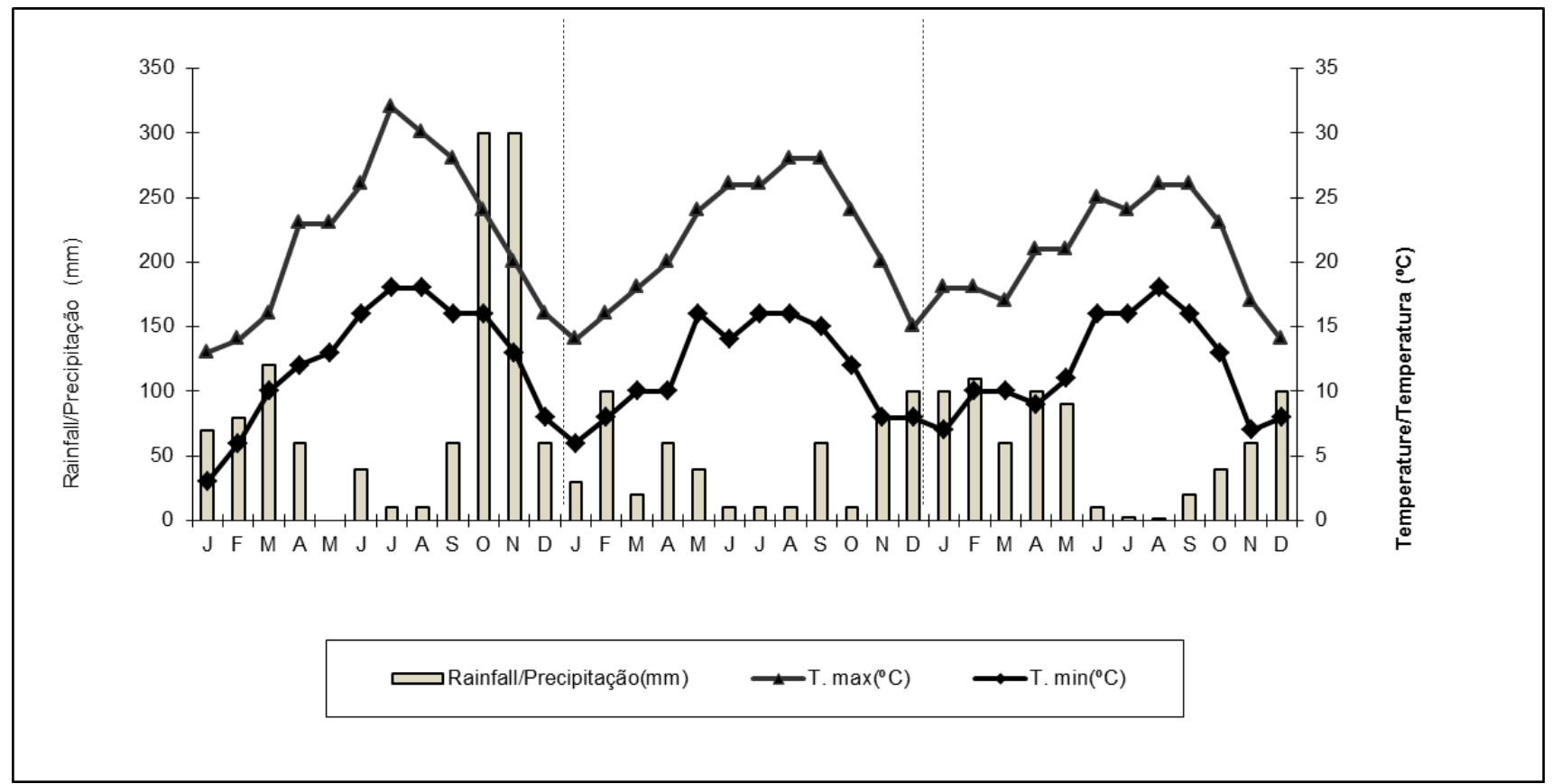

Figure 1/ Figura 1 - Climatic data obtained from the Instituto Português do Mar e da Atmosfera for the years 2006, 2007, and 2008. The values represent monthly averages.

Dados climáticos obtidos do Instituto Português do Mar e da Atmosfera para os anos 2006, 2007 e 2008. Os valores traduzem médias mensais. 
the study period. As regards temperature, the year 2006 emerges as the year with the lowest minimum temperature and the highest maximum temperature. In terms of rainfall, the year 2006 was the wettest of the three, but the year 2008 presents a distinguishing particularity; during this year it rained consistently and evenly throughout the winter and spring months until May, with atypically high rainfall in April and May. The results obtained in the year 2008 seem to indicate that the rainfall was the climatic factor that had the most effect on the essential oils content, both for the fruit and for the leaf, though with opposite outcomes. The essential oils content in the fruit was the lowest observed, likely through inhibition of their aromatic precursors triggered by draught stress which did not occur in 2008, thus resulting in low oils content in the fruit. Surprisingly, quite the opposite effect was observed on the oils content of the leaf, which clearly shows a rise in its value. This may indicatethat, the aromatic precursors for the leaf and for the fruit are not formed, within the plant at the same time.

\section{Essential Oils Composition}

Tables 2, and 3, and Figures 2, and 3 show the composition of the essential oils extracted from leaves and fruits of Myrtus communis L. collected during the three years in study. These results show that the principal components of Portuguese myrtle essential oils are $\alpha$-pinene, limonene $+1,8$ cineole, linalool and myrtenyl acetate. The results were statistically analysed applying oneway and two-way ANOVA to major components. The result revealed that for the major components the compositions are significantly different $(p<0.001)$ for the three years in study for both leaves and fruits.

Thirty-five compounds were identified in the leaves, representing $94.4 \%-96.8 \%$ of the total essential oils. The results show that the leaves composition wasdominated by hydrocarbon monoterpene compounds in the first two years of study. It can be observed that the content in monoterpenes decreased slightly from the first to the second year and then markedly in the third year, accompanying the changes that can be seen in the content of $\alpha$-pinene, which is the major monoterpene present in the oils. The results for alcohols composition show a slight decrease from 2006 to 2007 followed by an increase on alcohol content in 2008, this behaviour being closely followed by linalool, the main alcohol present in myrtle essential oils. As to the esters, their content increased steadily over the three years period, mainly due to the increase in myrtenyl acetate content.

With regard to the fruit, thirty-seven compounds were identified, representing $86.8 \%-93.9 \%$ of the total es-
A figura 1 mostra os dados climáticos para os três anos em análise. Algumas diferenças podem ser observadas entre os três anos. No que diz respeito à temperatura, $\mathrm{o}$ ano de 2006 surge como o ano com a mais baixa temperatura mínima e a temperatura máxima mais elevada. Em termos de precipitação, o ano de 2006 foi o mais chuvoso dos três, mas o ano de 2008 apresentou uma particularidade: durante este ano choveu de forma consistente e uniforme ao longo dos meses de inverno e primavera até maio, com chuvas atípicamente elevadas em abril e maio. Os resultados obtidos para o ano de 2008 parecem indicar que a precipitação tenha sido o fator climático que mais afetou o teor dos óleos essenciais nos frutos e nas folhas embora com resultados opostos. O teor dos óleos essenciais obtido para os frutos, nesse ano, foi o menor valor observado, provavelmente devido à inibição dos seus precursores do aroma que são desencadeados pelo stress da seca, que em 2008 não ocorreu, resultando num baixo teor dos óleos essenciais nos frutos. Surpreendentemente, observou-se o efeito contrário no teor dos óleos essenciais das folhas, em 2008, cujo valor registou uma clara subida. Isto, provavelmente, mostra que, na planta, os precursores de aroma para a folha e para o fruto, não são formados ao mesmo tempo

\section{Composição do Óleo Essencial}

Nas tabelas 2 e 3, e nas figuras 2 e 3 apresentam-se as composições dos óleos essenciais obtidos das folhas e frutos da murta colhida durante os três anos em estudo. Estes resultados mostram que os componentes principais dos óleos essenciais de murta colhida em Portugal são $\alpha$-pineno, limoneno $+1,8$ cineol, linalol e acetato de mirtenilo. Os resultados foram analisados estatisticamente, aplicando a ANOVA simples e de dois fatores aos componentes principais. O resultado revelou que, para os componentes principais, tanto para as folhas como para os frutos, as composições são significativamente diferentes $(p<0,001)$ para os três anos em estudo. Trinta e cinco compostos foram identificados nas folhas, o que representa $94,4 \%$ a $96,8 \%$ dos componentes identificados nos óleos essenciais. Os resultados mostram que os monoterpenos predominam na composição das folhas durante os dois primeiros anos. Pode observar-se que o teor de monoterpenos diminuiu ligeiramente do primeiro para o segundo ano e, em seguida, acentuadamente para o terceiro ano, acompanhando a variação que se observa no teor de $\alpha$-pineno, que é o principal monoterpeno presente nos óleos. Os resultados mostram ainda que a composição em álcoois apresenta uma ligeira diminuição de 2006 para 2007 seguida por um aumento no teor de álcool em 2008, sendo este compor- 
Table 2 / Tabela 2 - Myrtle leaves essential oils composition for the three years studied. Composição dos óleos essenciais das folhas de murta para os três anos de colheita.

\begin{tabular}{|c|c|c|c|c|c|}
\hline \multirow{2}{*}{ Components $^{\mathrm{a}} /$ Componentes $^{\mathrm{a}}$} & \multirow{2}{*}{$\mathbf{L R I}^{\mathbf{b}}$} & \multirow{2}{*}{$\begin{array}{l}\text { Ident.Method }^{\mathrm{c}} / \\
\text { Método Ident. }^{\mathrm{c}}\end{array}$} & \multicolumn{3}{|c|}{ Composition (\%) \pm SD $^{\mathrm{e}} /$ Composição $(\%) \pm \mathrm{DP}^{\mathrm{e}}$} \\
\hline & & & 2006 & 2007 & 2008 \\
\hline${\text { Tricyclene } / \text { Tricicleno }^{\mathrm{d}}}$ & 908 & MS, LRI & $0,08 \pm 0,00$ & $0,17 \pm 0,01$ & $0,12 \pm 0,01$ \\
\hline$\alpha$-Thujene/ $\alpha$-Tujeno ${ }^{\mathrm{d}}$ & 917 & MS, LRI & $0,10 \pm 0,00$ & $0,03 \pm 0,00$ & $0,03 \pm 0,00$ \\
\hline$\alpha$-Pinene/ $\alpha$-Pineno & 921 & MS, LRI, std & $21,50 \pm 0,10$ & $15,52 \pm 0,11$ & $5,32 \pm 0,07$ \\
\hline Camphene/ Canfeno $^{\mathrm{d}}$ & 937 & MS, LRI & $0,04 \pm 0,00$ & $0,04 \pm 0,00$ & $0,02 \pm 0,00$ \\
\hline$\beta$-Pinene $/ \beta$-Pineno & 963 & MS, LRI, std & $0,26 \pm 0,00$ & $0,14 \pm 0,00$ & $0,08 \pm 0,01$ \\
\hline Myrcene /Mirceno & 986 & MS, LRI, std & $0,14 \pm 0,00$ & $0,06 \pm 0,00$ & $0,07 \pm 0,00$ \\
\hline$\delta$-3-Carene $/ \delta$-3-Careno ${ }^{\mathrm{d}}$ & 998 & MS, LRI & $0,05 \pm 0,00$ & $0,32 \pm 0,01$ & $0,28 \pm 0,01$ \\
\hline$\alpha$-Terpinene $/ \alpha$-Terpineno & 1003 & MS, LRI, std & $0,11 \pm 0,01$ & $0,01 \pm 0,00$ & $0,02 \pm 0,00$ \\
\hline p-Cymene /p-Cimeno & 1015 & MS, LRI, std & $0,03 \pm 0,00$ & $0,05 \pm 0,00$ & $0,05 \pm 0,00$ \\
\hline $\begin{array}{l}\text { Limonene }+1,8 \text {-Cineole/ } \\
\text { Limoneno+1,8-Cineol }\end{array}$ & 1022 & MS, LRI, std & $39,45 \pm 0,10$ & $36,50 \pm 0,21$ & $25 \pm 1$ \\
\hline${\mathrm{o}-C y m e n e / \mathrm{o}-\mathrm{Cimeno}^{\mathrm{d}}}^{\mathrm{d}}$ & 1036 & MS, LRI & $0,02 \pm 0,00$ & $0,05 \pm 0,01$ & $0,06 \pm 0,01$ \\
\hline$\gamma$-Terpinene $/ \gamma$-Terpineno & 1043 & MS, LRI, std & $0,11 \pm 0,00$ & $0,17 \pm 0,00$ & $0,10 \pm 0,00$ \\
\hline Linalool oxide/Óxido de Linalol & 1051 & MS, LRI, std & $0,30 \pm 0,00$ & $0,04 \pm 0,00$ & $0,05 \pm 0,02$ \\
\hline$\alpha$-Terpinolene $/ \alpha$-Terpinoleno ${ }^{\mathrm{d}}$ & 1081 & MS, LRI, std & $0,25 \pm 0,01$ & $0,16 \pm 0,00$ & $0,09 \pm 0,00$ \\
\hline Linalool/Linalol & 1097 & MS, LRI, std & $6,19 \pm 0,03$ & $6,55 \pm 0,04$ & $16,33 \pm 0,14$ \\
\hline Fenchol /Fenchol & 1100 & MS, LRI, std & $0,52 \pm 0,02$ & $0,65 \pm 0,06$ & $0,80 \pm 0,07$ \\
\hline Trans pinocarveol/Trans pinocarveol $^{\mathrm{d}}$ & 1129 & MS, LRI & $0,04 \pm 0,00$ & $0,05 \pm 0,00$ & $0,04 \pm 0,01$ \\
\hline Borneol /Borneol & 1160 & MS, LRI, std & $0,14 \pm 0,00$ & $0,07 \pm 0,00$ & $0,05 \pm 0,02$ \\
\hline Terpinen-4-ol /Terpineno-4-ol & 1169 & MS, LRI, std & $0,29 \pm 0,00$ & $0,21 \pm 0,01$ & $0,17 \pm 0,01$ \\
\hline$\alpha$-Terpineol $/ \alpha$-Terpineol & 1184 & MS, LRI, std & $5,15 \pm 0,02$ & $3,44 \pm 0,12$ & $2,9 \pm 0,3$ \\
\hline Myrtenol /Mirtenol & 1189 & MS, LRI, std & $0,32 \pm 0,00$ & $1,10 \pm 0,01$ & $1,24 \pm 0,18$ \\
\hline Geraniol /Geraniol & 1255 & MS, LRI, std & $0,89 \pm 0,00$ & $0,81 \pm 0,01$ & $0,94 \pm 0,06$ \\
\hline Borneol acetate/Acetato de Borneol & 1282 & MS, LRI, std & $0,02 \pm 0,00$ & $0,02 \pm 0,00$ & $0,05 \pm 0,02$ \\
\hline $\begin{array}{l}\text { Trans-pinocarvyl acetate/Acetato de } \\
\text { transpinocarvilo }\end{array}$ & 1292 & MS, LRI & $0,16 \pm 0,01$ & $0,39 \pm 0,03$ & $0,54 \pm 0,01$ \\
\hline Myrtenyl acetate /Acetato de mirtenilo & 1322 & MS, LRI, std & $7,40 \pm 0,02$ & $22,4 \pm 0,4$ & $36 \pm 1$ \\
\hline $\begin{array}{c}\alpha \text {-Terpenyl acetate/Acetato de } \alpha \text { - } \\
\text { terpenilo }\end{array}$ & 1337 & MS, LRI, std & $0,22 \pm 0,00$ & $0,21 \pm 0,01$ & $0,05 \pm 0,02$ \\
\hline Eugenol/Eugenol $^{\mathrm{d}}$ & 1343 & MS, LRI, std & $1,54 \pm 0,01$ & $0,54 \pm 0,01$ & $0,16 \pm 0,02$ \\
\hline Neryl acetate/Acetato de nerilo & 1364 & MS, LRI, std & $0,12 \pm 0,02$ & $0,06 \pm 0,00$ & $0,0 \underline{4} \pm 0,01$ \\
\hline Geranyl acetate/Acetato de geranilo & 1377 & MS, LRI, std & $0,11 \pm 0,00$ & $0,18 \pm 0,01$ & $0,33 \pm 0,00$ \\
\hline Methyleugenol/Metileugenol $^{\mathrm{d}}$ & 1383 & MS, LRI, std & $4,26 \pm 0,02$ & $2,68 \pm 0,01$ & $1,76 \pm 0,07$ \\
\hline$\beta$-Caryophyllene/ $\beta$-Cariofileno & 1404 & MS, LRI, std & $2,18 \pm 0,01$ & $2,42 \pm 0,06$ & $2,11 \pm 0,17$ \\
\hline$\alpha$-Humulene/ $\alpha$-Humuleno & 1442 & MS, LRI, std & $0,84 \pm 0,01$ & $0,22 \pm 0,03$ & $0,29 \pm 0,14$ \\
\hline $\begin{array}{l}\text { Geranyl isobutyrate/Isobutirato de } \\
\text { geranilo }^{\mathrm{d}}\end{array}$ & 1513 & MS, LRI, std & $0,98 \pm 0,01$ & $0,67 \pm 0,09$ & $0,54 \pm 0,09$ \\
\hline $\begin{array}{l}\text { Caryophyllene oxide/Óxido de } \\
\text { cariofileno }\end{array}$ & 1573 & MS, LRI, std & $0,27 \pm 0,02$ & $0,24 \pm 0,02$ & $0,21 \pm 0,02$ \\
\hline Humulene oxide/Óxido de humuleno $^{\mathrm{d}}$ & 1599 & MS, LRI, std & $0,31 \pm 0,01$ & $0,36 \pm 0,03$ & $0,23 \pm 0,02$ \\
\hline Monoterpenes / Monoterpenos & & & 62,1 & 53,2 & 31,3 \\
\hline Alcohols / Álcoois & & & 15,1 & 13,4 & 22,6 \\
\hline Esters / Ésteres & & & 9,0 & 23,9 & 37,5 \\
\hline Ethers / Éteres & & & 4,3 & 2,7 & 1,8 \\
\hline Sesquiterpenes / Sesquiterpenos & & & 3,3 & 2,9 & 2,6 \\
\hline Oxides / Óxidos & & & 0,9 & 0,6 & 0,5 \\
\hline $\begin{array}{l}\text { Identified Compounds / Compostos } \\
\text { identificados }\end{array}$ & & & 94,4 & 96,8 & 96,3 \\
\hline
\end{tabular}

a- Compounds are listed in order of their elution from a DB-5MS column: b- Linear retention indices as determined on a DB-5MS column using a homologous series of n-alkanes; c- Methods of identification: MS, by comparison of the mass spectrum with those of the computer mass libraries; LRI, by comparison of LRI with those from the literature; std, by injection of the authentic sample;d- Tentatively identified: according with mass spectrum and through comparison of LRI with the linear retention time; e- Standard deviation.

a- Compostos são listados pela ordem da sua eluição a partir de uma coluna DB-5MS; b- Índices de retenção linear determinados numa coluna DB-5MS utilizando uma série homóloga de n-alcanos; c- Métodos de identificação: MS, por comparação do espetro de massa com os existentes nas bibliotecas informáticas; LRI- por comparação dos LRI com os da literatura; std - por injeção de padrões; dTentativamente identificados de acordo com o espetro de massa e por comparação dos LRI com os tempos de retenção linear; e - Desviopadrão. 
Table 3/ Tabela 3 - Myrtle berries essential oils composition for the three years studied. Composição dos óleos essenciais das bagas de murta para os três anos de colheita.

\begin{tabular}{|c|c|c|c|c|c|}
\hline \multirow[t]{2}{*}{ Components $^{\mathrm{a}} /$ Componentes $^{\mathrm{a}}$} & \multirow[t]{2}{*}{ LRI $^{\mathrm{b}}$} & \multirow{2}{*}{$\begin{array}{l}\text { Ident.Method }{ }^{\mathrm{c} /} \\
\text { Método Ident. }^{\mathrm{c}}\end{array}$} & \multicolumn{3}{|c|}{ Composition (\%) $\pm \mathrm{SD}^{\mathrm{e}} /$ Composição $(\%) \pm \mathrm{DP}^{\mathrm{e}}$} \\
\hline & & & 2006 & 2007 & 2008 \\
\hline Tricyclene Tricicleno $^{\mathrm{d}}$ & 911 & MS, LRI & $0.05 \pm 0.00$ & $0.03 \pm 0.01$ & $0.02 \pm 0.00$ \\
\hline$\alpha$-Thujene/ $\alpha$-Tujeno ${ }^{\mathrm{d}}$ & 919 & MS, LRI & $0.04 \pm 0.00$ & $0.07 \pm 0.01$ & $0.03 \pm 0.00$ \\
\hline$\alpha$-Pinene $/ \alpha$-Pineno & 923 & MS, LRI, std & $9.65 \pm 0.02$ & $7.31 \pm 0.43$ & $3.54 \pm 0.12$ \\
\hline Camphene/ Canfeno $^{\mathrm{d}}$ & 943 & MS, LRI & $0.01 \pm 0.00$ & $0.01 \pm 0.00$ & $0.02 \pm 0.00$ \\
\hline$\beta$-Pinene/ $\beta$-Pineno & 965 & MS, LRI, std & $0.17 \pm 0.00$ & $0.14 \pm 0.04$ & $0.09 \pm 0.00$ \\
\hline Myrcene/Mirceno & 992 & MS, LRI, std & $0.07 \pm 0.01$ & $0.12 \pm 0.04$ & $0.06 \pm 0.001$ \\
\hline$\delta$-3-Carene $/ \delta-3$-Careno ${ }^{\mathrm{d}}$ & 1003 & MS, LRI & $0.02 \pm 0.01$ & $0.05 \pm 0.02$ & $0.02 \pm 0.01$ \\
\hline$\alpha$-Terpinene $/ \alpha$-Terpineno & 1010 & MS, LRI, std & $0.13 \pm 0.01$ & $0.32 \pm 0.03$ & $0.13 \pm 0.03$ \\
\hline p-Cymene /p-Cimeno & 1020 & MS, LRI, std & $0.11 \pm 0.00$ & $0.03 \pm 0.01$ & $0.05 \pm 0.00$ \\
\hline $\begin{array}{l}\text { Limonene+1,8-Cineole/ } \\
\text { Limoneno+1,8-Cineol }\end{array}$ & 1025 & MS, LRI, std & $25.28 \pm 0.09$ & $21.65 \pm 0.68$ & $12.83 \pm 0.27$ \\
\hline o-Cymene/o-Cimeno ${ }^{\mathrm{d}}$ & 1040 & MS, LRI & & $0.02 \pm 0.01$ & $0.04 \pm 0.01$ \\
\hline$\gamma$-Terpinene $/ \gamma$-Terpineno & 1053 & MS, LRI, std & $0.16 \pm 0.01$ & $0.33 \pm 0.03$ & $0.09 \pm 0.00$ \\
\hline Linalool oxide/Óxido de Linalol & 1071 & MS, LRI, std & $0.02 \pm 0.00$ & $0.07 \pm 0.04$ & $0.03 \pm 0.02$ \\
\hline$\alpha$-Terpinolene $/ \alpha$-Terpinoleno ${ }^{\mathrm{d}}$ & 1083 & MS, LRI & $0.07 \pm 0.01$ & $0.23 \pm 0.03$ & $0.05 \pm 0.00$ \\
\hline Linalool /Linalol & 1098 & MS, LRI, std & $6.56 \pm 0.07$ & $3.39 \pm 0.19$ & $12.52 \pm 0.24$ \\
\hline Fenchol/Fenchol & 1102 & MS, LRI, std & $0.65 \pm 0.03$ & $0.47 \pm 0.02$ & $0.63 \pm 0.00$ \\
\hline Trans pinocarveol/Trans pinocarveol ${ }^{\mathrm{d}}$ & 1130 & MS, LRI & $0.17 \pm 0.04$ & $0.16 \pm 0.03$ & $0.09 \pm 0.01$ \\
\hline Borneol /Borneol & 1162 & MS, LRI, std & $0.10 \pm 0.01$ & $0.15 \pm 0.02$ & $0.07 \pm 0.01$ \\
\hline Terpinen-4-ol /Terpineno-4-ol & 1171 & MS, LRI, std & $0.26 \pm 0.00$ & $0.50 \pm 0.01$ & $0.23 \pm 0.01$ \\
\hline$\alpha$-Terpineol $/ \alpha$-Terpineol & 1186 & MS, LRI, std & $4.01 \pm 0.01$ & $3.60 \pm 0.22$ & $2.89 \pm 0.17$ \\
\hline Myrtenol /Mirtenol & 1191 & MS, LRI, std & $3.24 \pm 0.02$ & $3.42 \pm 0.19$ & $3.51 \pm 0.20$ \\
\hline Geraniol /Geraniol & 1255 & MS, LRI, std & $0.93 \pm 0.00$ & $0.87 \pm 0.03$ & $0.75 \pm 0.04$ \\
\hline Borneol acetate/Acetato de Borneol & 1280 & MS, LRI, std & $0.06 \pm 0.07$ & $0.04 \pm 0.01$ & $0.04 \pm 0.00$ \\
\hline $\begin{array}{l}\text { Trans-pinocarvyl acetate/Acetato de } \\
\text { transpinocarvilo }\end{array}$ & 1294 & MS, LRI & $0.64 \pm 0.00$ & $0.65 \pm 0.06$ & $0.75 \pm 0.02$ \\
\hline Myrtenyl acetate /Acetato de mirtenilo & 1327 & MS, LRI, std & $32.86 \pm 0.21$ & $30.87 \pm 0.35$ & $39.90 \pm 1.37$ \\
\hline $\begin{array}{c}\alpha \text {-Terpenyl acetate/Acetato de } \alpha \text { - } \\
\text { terpenilo }\end{array}$ & 1338 & MS, LRI & $0.54 \pm 0.004$ & $0.70 \pm 0.02$ & $0.61 \pm 0.02$ \\
\hline Eugenol/Eugenol ${ }^{\mathrm{d}}$ & 1346 & MS, LRI & $0.26 \pm 0.00$ & $1.81 \pm 0.02$ & $0.11 \pm 0.02$ \\
\hline Neryl acetate/Acetato de nerilo & 1369 & MS, LRI, std & $0.07 \pm 0.00$ & $0.20 \pm 0.01$ & $0.06 \pm 0.01$ \\
\hline Geranyl acetate/Acetato de geranilo & 1379 & MS, LRI, std & $0.19 \pm 0.00$ & $0.24 \pm 0.02$ & $0.32 \pm 0.00$ \\
\hline Methyleugenol/Metileugenol $^{\mathrm{d}}$ & 1384 & MS, LRI & $3.50 \pm 0.03$ & $4.18 \pm 0.06$ & $2.7^{\prime} \pm 0.01$ \\
\hline$\beta$-Caryophyllene/ $\beta$-Cariofileno & 1404 & MS, LRI, std & $1.79 \pm 0.04$ & $3.59 \pm 0.20$ & $2.30 \pm 0.20$ \\
\hline$\alpha$-Humulene/ $\alpha$-Humuleno & 1445 & MS, LRI, std & $0.34 \pm 0.00$ & $0.33 \pm 0.01$ & $0.48 \pm 0.04$ \\
\hline$\beta$-Selinene ${ }^{d} / \beta$-Selineno ${ }^{d}$ & 1478 & MS, LRI & $0.13 \pm 0.00$ & $0.30 \pm 0.18$ & $0.11 \pm 0.01$ \\
\hline$\alpha$-Selinene ${ }^{d} / \alpha$-Selineno ${ }^{d}$ & 1487 & MS, LRI & $0.12 \pm 0.01$ & $0.20 \pm 0.05$ & $0.11 \pm 0.01$ \\
\hline $\begin{array}{c}\text { Geranyl isobutyrate/Isobutirato de } \\
\text { geranilo }^{\mathrm{d}}\end{array}$ & 1513 & MS, LRI & $0.32 \pm 0.01$ & $0.55 \pm 0.16$ & $0.32 \pm 0.07$ \\
\hline $\begin{array}{l}\text { Caryophyllene oxide/Óxido de } \\
\text { cariofileno }\end{array}$ & 1574 & MS, LRI, std & $0.67 \pm 0.01$ & $0.62 \pm 0.05$ & $0.83 \pm 0.03$ \\
\hline Humulene oxide/Óxido de humuleno $^{\mathrm{d}}$ & 1601 & MS, LRI, std & $0.61 \pm 0.03$ & $0.74 \pm 0.19$ & $0.65 \pm 0.05$ \\
\hline Monoterpenes / Monoterpenos & & & 35.8 & 30.3 & 17.0 \\
\hline Alcohols / Álcoois & & & 16.2 & 14.37 & 20.7 \\
\hline Esters / Ésteres & & & 34.7 & 33.25 & 42.0 \\
\hline Ethers / Éteres & & & 3.5 & 4.18 & 2.7 \\
\hline Sesquiterpenes / Sesquiterpenos & & & 3.0 & 5.03 & 3.8 \\
\hline Oxides / Óxidos & & & 1.3 & 1.43 & 1.5 \\
\hline $\begin{array}{c}\text { Identified Compounds / Compostos } \\
\text { identificados } \\
\end{array}$ & & & 93.9 & 88.0 & 86.8 \\
\hline
\end{tabular}

a- Compounds are listed in order of their elution from a DB-5MS column: b- Linear retention indices as determined on a DB-5MS column using a homologous series of n-alkanes; c- Methods of identification: MS, by comparison of the mass spectrum with those of the computer mass libraries; LRI, by comparison of LRI with those from the literature; std, by injection of the authentic sample;d- Tentatively identified: according with mass spectrum and through comparison of LRI with the linear retention time; e- Standard deviation.

a- Compostos são listados pela ordem da sua eluição a partir de uma coluna DB-5MS; b- Índices de retenção linear determinados numa coluna DB-5MS utilizando uma série homóloga de n-alcanos; c- Métodos de identificação: MS, por comparação do espetro de massa com os existentes nas bibliotecas informáticas; LRI- por comparação dos LRI com os da literatura; std - por injeção de padrões; d- Tentativamente identificados de acordo com o espetro de massa e por comparação dos LRI com os tempos de retenção linear; e - Desvio-padrão. 


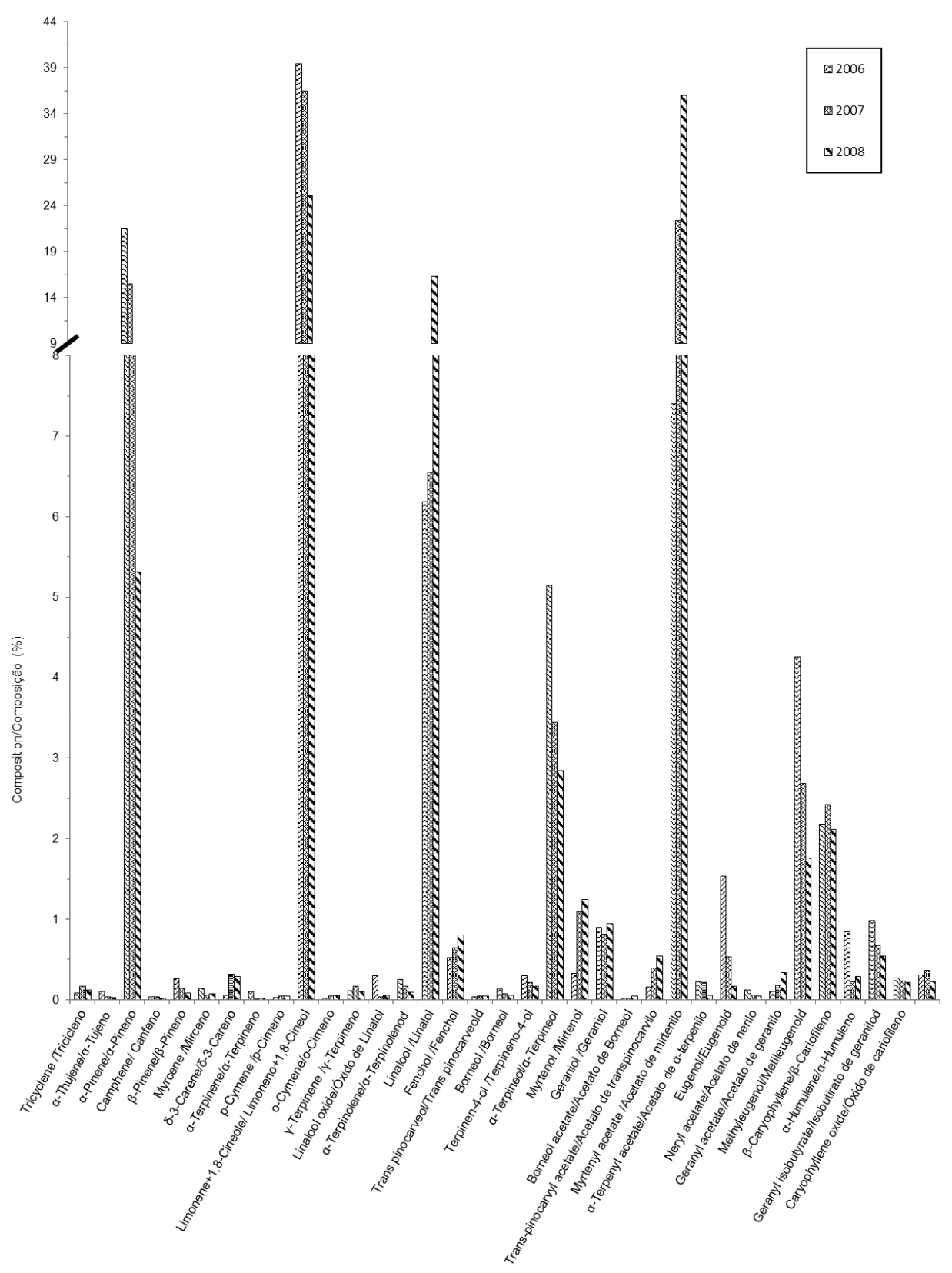

Figure 2/ Figura 2 - Composition of leaves essential oils of Portuguese myrtle harvested in July for the years in study.

Composição dos óleos essenciais das folhas de murta recolhidas no mês de julho dos anos em estudo. 


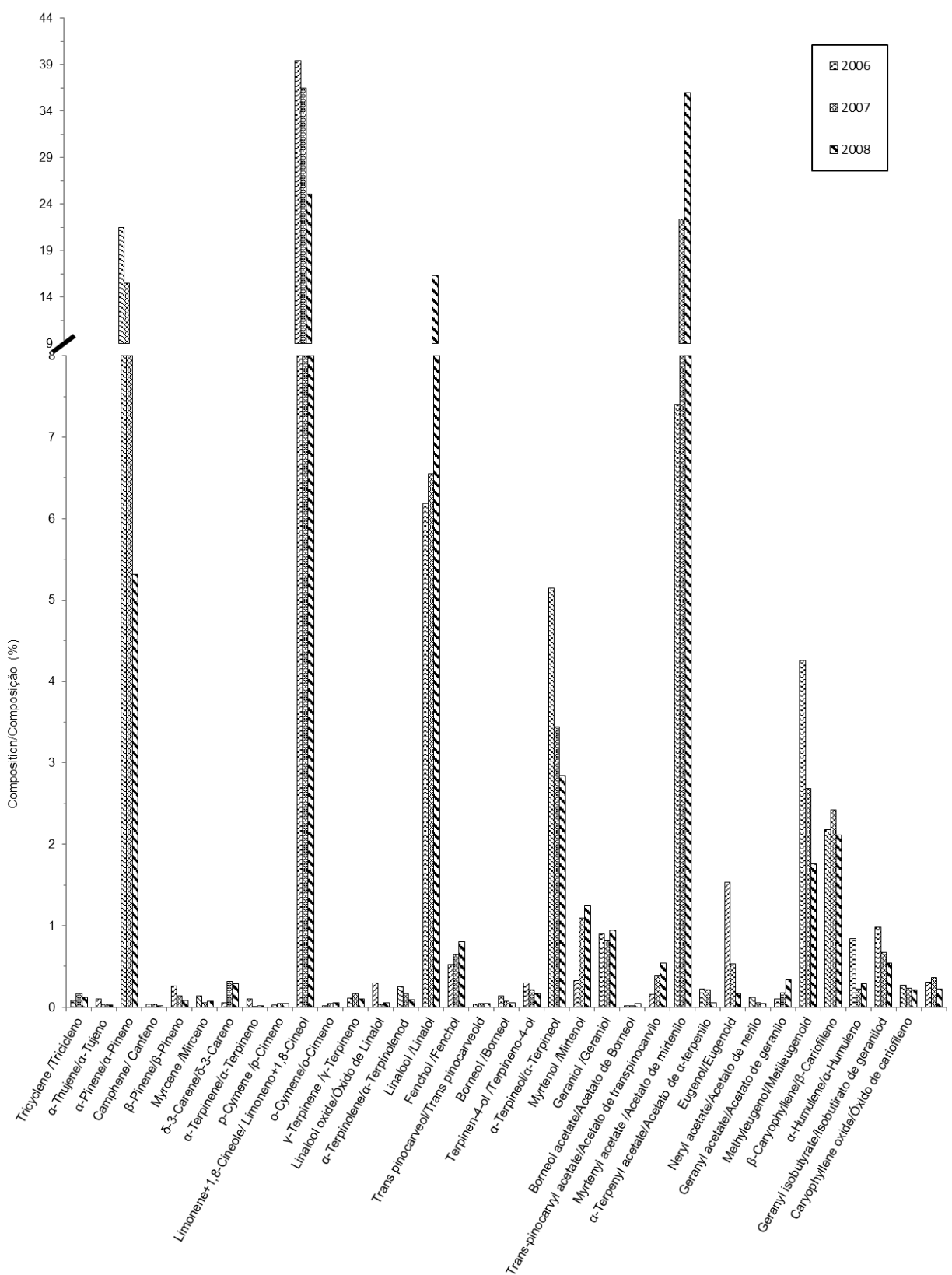

Figure 3/ Figura 3 - Composition of berries essential oils of Portuguese myrtle collected in October for the years in study

Composição dos óleos essenciais das bagas de murta recolhidas no mês de outubro dos anos em estudo. 
sential oils. In the first two years of study, the results show that the composition was dominated by monoterpenes and esters compounds, with only small variations from one year to the other. However, in the third year, 2008, the trend changed. While the esters show a moderate increase, due to the increase in myrtenyl acetate, the monoterpenes decreased sharply, mainly owing to the accentuated decrease in both $\alpha$-pinene and limonene $+1,8$-cineole contents. The results for alcohols composition show a very slight decrease from 2006 to 2007, followed by an increase in alcohol content in 2008 . This behaviour closely followed that of the linalool, the main alcohol present in myrtle essential oils, which underwent a sharp increase in 2008. The content of esters increased steadily over the three years period, mainly owing to the increase in myrtenyl acetate content.

The results seem to indicate that the long (January to May) and persistent rainfall period in 2008 caused a decrease in the content of $\alpha$-pinene and limonene $+1,8$ cineole and an increase in the myrtenyl acetate content in the both leaves and the berries.

The temperature also affected the essential oils composition. During the ripening period, the year when the maximum temperature recorded was higher (2008) was also the year when the contents in $\alpha$-pinene and limonene $+1,8$-cineole was higher, both from the leaves and the fruit. Nevertheless, a direct relationship with the temperature cannot be observed regarding the myrtenyl acetate content, unlike that for precipitation.

\section{Conclusion}

In conclusion, it is possible to say that climatic conditions affect the composition of the essential oils in both the leaves and berries of Portuguese myrtle. The essential oils are substances produced by the plant as part of a response mechanism to stress, which can are most often water or temperature dependent. Customarily, these are the main factors that affect the vegetative cycle of plants. The results of this work indicate that the yield and composition of the essential oils show a stronger dependence on precipitation, which may indicate that the production of essential oils by the plant is triggered by water-related stress when the precipitation occurs at tamento seguido pelo linalol, o principal álcool presente nos óleos essenciais da murta. Quanto aos ésteres, o seu teor sofreu um aumento constante durante o período de 3 anos, principalmente devido ao aumento do teor de acetato de mirtenilo.

Em relação aos frutos, trinta sete compostos foram identificados, o que representa $86,8 \%$ a $93,9 \%$ dos componentes identificados nos óleos essenciais. Nos primeiros dois anos, os resultados mostram que na composição predominam os monoterpenos e ésteres, com apenas pequenas variações de um ano para o outro, mas, no terceiro ano, 2008, a tendência mudou. Embora os ésteres apresentem um aumento moderado, devido ao aumento de acetato de mirtenilo, os monoterpenos diminuíram acentuadamente, principalmente devido ao decréscimo acentuado no teor de $\alpha$-pineno e de limoneno+1,8-cineol. Os resultados para a composição em álcoois mostram uma diminuição muito ligeira do ano 2006 para o de 2007, seguida por um aumento do teor de álcool em 2008, sendo este comportamento seguido pelo linalol, o principal álcool presente nos óleos essenciais de murta, que sofreu um forte aumento em 2008. O teor de ésteres aumentou regularmente durante o período de 3 anos, principalmente devido ao aumento do teor de acetato de mirtenilo. Os resultados parecem apontar para que o longo (janeiro a maio) e persistente período de precipitação em 2008 tenha causado um decréscimo no conteúdo de $\alpha$-pineno e limoneno $+1,8$ -cineol e um aumento do teor de acetato de mirtenilo tanto nas folhas como nas bagas.

A temperatura também parece afetar a composição dos óleos essenciais. Durante o período de amadurecimento, o ano em que se registou a temperatura máxima mais elevada (2008), foi também o ano em que o teor em $\alpha$-pineno e limoneno $+1,8$-cineol foi mais elevado, tanto na folha como no fruto. No entanto, no teor de acetato de mirtenilo não se observa uma relação direta com a temperatura, ao contrário do que acontece relativamente à precipitação.

\section{Conclusão}

Em conclusão, é possível afirmar que as condições climáticas afetam a composição dos óleos essenciais nas folhas e nos frutos da murta portuguesa. Os óleos essenciais são substâncias produzidas pela planta como parte de um mecanismo de resposta ao stress, que pode ser dependente da precipitação ou da temperatura. Geralmente, estes são os principais fatores que afetam o ciclo vegetativo das plantas. Os resultados deste trabalho indicam que a produção e composição dos óleos essenciais mostram uma forte dependência da precipitação, o que pode indicar que a produção de óleos essenciais, pela planta, é desencadeada pelo stress relacionado com 
the time of the vegetative cycle that the aromatic precursors are being formed.

\section{Conflict of interests}

The authors declare that there are no financial and/or personal relationships that could be viewed as presenting a potential conflict of interests. a precipitação quando esta acontece na fase do ciclo vegetativo em que se formam os percursores do aroma.

\section{Conflito de Interesses}

Os autores declaram não existir qualquer relação pessoal ou financeira que possa ser entendida como representando um potencial conflito de interesses. 


\section{References / Referências}

[1] Wannes A, Mhamdi B, Marzouk B. GC Comparative Analysis of Leaf Essential Oils from Two Myrtle Varieties at Different Phenological Stages. Chromatographia 2009; 69:145150.

[2] Elfellah, MS, Akhter M. H., Khan, MT. Antihyperglycaemic effect of an extract of Myrtus communis in Streptozotocin-induced diabetes in mice. J. Ethnopharmacology 1984; 11:275-281.

[3] Pereira P, Bernardo-Gil MG, Cebola MJ, Mauricio E, Romano A. Supercritical fluid extracts with antioxidant and antimicrobial activities from myrtle (Myrtus communis L.) leaves. Response surface optimization. J. Supercrit. Fluids 2013; 83: 57-64.

[4] Pereira PC, Cebola MJ, Bernardo-Gil M G. Evolution of the Yields and Composition of Essential Oil from Portuguese Myrtle (Myrtus comunis L.) through the Vegetative. Cycle. Molecules 2009, 14:3094-3105.

[5] Tuberoso C, Barra A, Angioni A, Sarritzu E, Pirisi F. Chemical composition of volatiles in Sardinian Myrtle (Myrtus communis L.) alcoholic extracts and essential oils. J. Agric. Food Chem. 2006; 54:1420-1426.
[6] Scora RW. Essential leaf oil variability in green, variegated and albino foliage of Myrtus communis. Phytochemistry 1973; 12:153-155.

[7] Akin M, Aktumsek A, Nostro A. Antibacterial activity and composition of the essential oils of Eucalyptus camaldulensis Dehn. and Myrtus communis L. growing in Northern Cyprus. African J. of Biotechnology 2010; 9 (4): 531-535.

[8] Apostolova P, Yaneva I. Antioxidative Defence in Winter Wheat Plants During Early Cold Acclimation. Gen. Appl. Plant Physiology. 2006; Special issue:101-108.

[9] Larcher W. Temperature stress and survival ability of Mediterranean sclerophyllous. Plant Biosystems 2000; 134 (3):279-295.

[10] Fadda, A., Mulas, M. Chemical changes during myrtle (Myrtus communis L.) fruit development and ripening. Scientia Horticulturae 2010; 125:477-485.

[11] Asllani U. Chemical Composition of Albanian Myrtle Oil. J. Essen. Oil Res 2000; 12: 140-142.
[12] Jerkovic I., Radonic A, Borcic I. Comparative Study of Leaf, Fruit and Flower Essential Oil of Croatian Myrtus communis During a One Year Vegetative Cycle. J. Essen. Oil Res 2002; 14:266-270.

[13] Jamoussi B, Romdhane M, Abderraba A., Hassine B, Gadri A. Effect of harvest time on the yield and composition of Tunisian myrtle oils. J. Flavour and Fragrance 2005; 20:274-277.

[14] Zellner B, Bicchi C, Dugo P, Rubiolo P, Dugo G. Mondello, L.. Linear retention in gas chromatographic analysis: a review. Flavour and Fragrance J. 2008; 23:297-314.

[15] Babushok V, Zenkevich I. Retention Indices for most frequently reported essential Oil Compounds in GC. Chomatographia 2009; 69:257-269. 